\title{
De la comunicación para el desarrollo a la justicia ecosocial y el buen vivir
}

\author{
Alejandro BARRANQUERO*
}

Propuesto: 9 de marzo de 2012

Evaluado: 10 de abril de 2012

Aceptado: 17 de abril de 2012

(Abstracts y palabras clave al final del texto)

\section{UNA CIENCIA COLONIAL Y ECONOMICISTA}

Los estudios de comunicación para el desarrollo y para el cambio social cuentan con una dilatada trayectoria académica y práctica, especialmente en Latinoamérica y en el contexto anglosajón, hasta el punto de conformar una de las disciplinas más veteranas de las ciencias de la comunicación ${ }^{1}$. No obstante, desde la aparición de los primeros estudios científicos en EE.UU. su evolución se ha visto lastrada por un conjunto de limitaciones estructurales. Estas son fáciles de detectar en la práctica actual de los principales organismos y agencias mundiales de cooperación - FAO, UNESCO, BM, USAID, AECID-, que heredan una concepción fallida de los términos que componen la ecuación comunicación-para-el-desarrollo, a saber:

- Una perspectiva en exceso instrumental de la comunicación, concebida como simples medios o tecnologías siempre al servicio de algo - comunicación para-: salud, medioambiente, educación, ciudadanía; sin que se llegue a valorar la autonomía y potencial interdisciplinar de la comunicación para articular las diferentes áreas del conocimiento.

- Una óptica demasiado universalista y procedimental, en torno a los ideales de un desarrollo único - concebido desde Occidente- y del diseño y ejecución de metodologías exportables —edutainment, social marketing - en base a unos parámetros fácilmente mensurables -índices de desarrollo humano, indicadores de conectividad a las TIC-; una óptica que omite, por defecto, la reflexión situada y crítica desde la que debe de partir cualquier método transformador.

* Universidad Carlos III de Madrid abarranq@hum.uc3m.es

1 Los fundamentos teóricos de la comunicación para el desarrollo aparecen entre las tempranas preocupaciones de los padres fundadores de la disciplina -Lasswell, Berlo, Schramm, Lerner- desde finales de los 50 , vinculados al nacimiento de las primeras agencias y programas de cooperación: FAO, UNESCO, USAID, 
- Una visión orientada al desarrollo económico y acumulativo - teorías de la modernización - o, a nivel más extenso, al desarrollo androcéntrico - paradigma participativo- , pero, en uno y otro caso, centrada en el progreso del hombre y en sus posibilidades de intervención sobre el entorno natural.

En los últimos años están surgiendo profundos cuestionamientos a la visión lineal y mecanicista de estos programas. Algunas de los cuestionamientos parten de una deconstrucción y reelaboración del propio concepto de desarrollo primero, en base a etiquetas y añadidos - post-desarrollo, otro desarrollo, desarrollo humano, desarrollo integral, desarrollo sostenible, cambio social sostenible-; otras, las más avanzadas, decretan, en cambio, el acta de defunción definitiva al ideal del progreso o el cambio social, fijando su mirada en otras dimensiones: justicia social y ecológica, ciudadanía, empoderamiento, buen vivir, etc.

En este sentido, una de las revoluciones más importantes que ha vivido nuestro campo comenzó a gestarse en unas vitales reuniones en Bellagio (1997) y Cape Town $(1998)^{2}$, en las que algunos de los profesionales y teóricos más relevantes de la tan cuestionada comunicación para el desarrollo acordaron promover una nueva definición y orientación para la disciplina: la de la comunicación para el cambio social. El objetivo era desestimar la noción poscolonial y moderna de desarrollo, al tiempo que se definía un programa común para el nuevo siglo, basado en un enfoque dialógico, participativo y endógeno de la comunicación, y en la articulación de un cambio asentado en dinámicas comunitarias: "Comunicación para el cambio social es el proceso de diálogo público y privado a través del cual las personas definen quiénes son, cuáles son sus aspiraciones, qué es lo que necesitan y cómo pueden actuar colectivamente para alcanzar sus metas y mejorar sus vidas" (GrayFelder y Deane, 1999).

La propuesta de los expertos contribuyó a relanzar el debate y a revitalizar el campo a partir de la publicación de los primeros readers y antologías sobre la materia - Gumucio-Dagron, 2001; Gumucio y Tufte, 2006-, la celebración de importantes congresos a nivel mundial — World Congress on Communication for Development, Roma, $2006^{3}$ — o la articulación de programas de formación coordinados — Red de Universidades de Comunicación para el Cambio Social y el Desarrollo, 2005-. Sin embargo, más allá de los primeros alineamientos institucionales (Gray-Felder \& Deane, 1999), el concepto de comunicación para el cambio

etc. Paralelamente surge en Latinoamérica una perspectiva más crítica y participativa del área -Beltrán, Freire, Kaplún, Simpson-, vinculada a la experiencia de los movimientos sociales emancipatorios del continente: radios mineras, experiencias edu-comunicativas en Brasil (Paulo Freire), radio Sutatenza-ACPO en Colombia, etc.

2 Financiadas por la Rockefeller Foundation y a posteriori por el Consorcio de la Comunicación para el Cambio Social, en base al apoyo de esta fundación.

3 En este sentido, parece que la visión participativa de la comunicación para el cambio social amplía también su proyección en los grandes debates internacionales sobre el campo y en las agencias internacionales de cooperación. En este sentido, cabría destacar el rol de ciertas instituciones críticas en el Congreso Mundial de la Comunicación para el Desarrollo (WCCD), en Roma (2006), alentado conjuntamente por la FAO, el Banco Mundial y la Iniciativa de la Comunicación, "organizaciones que tienen diferente naturaleza y poco en común en su enfoque sobre el desarrollo" (Gumucio-Dagron, 2007). 
social no ha sido discutido en profundidad más que en algunos trabajos (Chaparro, 2009a, 2009b; Cimadevilla, 2004; Barranquero, 2011a), pero aún son escasos los cuestionamientos estructurales de lo que, a todas luces, se sigue mostrando como un concepto poco reflexivo, acrítico con sus fundamentos de partida y heredero, en buena medida, del programa originario: la comunicación para el desarrollo.

Las siguientes líneas están orientadas a problematizar acerca de la nueva configuración del campo en los últimos años, de acuerdo a planteamientos que nos aproximan a una teoría crítica de la cultura y el medioambiente como elementos interrelacionados y valor básico para cualquier concepción transformadora. Para ello se procederá, en primer lugar, a discutir las premisas sobre las que se asienta el paradigma dominante de la comunicación para el cambio social. En segunda instancia, se plantearán un conjunto de salidas a partir del diálogo interdisciplinar con diversas áreas del conocimiento que entroncan con el paradigma participativo en comunicación/desarrollo: educación ambiental, comunicación ambiental y Estudios Culturales Verdes. Por último, introduciremos dos nociones que pueden contribuir a guiar en lo próximo las nuevas aproximaciones a la materia: justicia ecosocial y buen vivir.

\section{LOS FUNDAMENTOS DE UN PARADIGMA INSOSTENIBLE}

La constitución del nuevo paradigma de la comunicación para el cambio social arrastra desde sus inicios un conjunto de errores y limitaciones sistémicos. Esto es fácil de comprobar con tan sólo descomponer los términos y la jerarquía que se da en el binomio comunicación-para-el cambio social, patente en algunos de los escritos fundacionales de la nueva perspectiva (Gray-Felder y Deane, 1999; GumucioDagron y Tufte, 2006):

\subsection{UNA COMUNICACIÓN INSTRUMENTAL Y ASISTENCIALISTA.}

Heredero de los primeros planteamientos modernizadores, el nuevo concepto de la comunicación para el cambio social sigue insistiendo en que la comunicación es una mera herramienta subsidiaria de los objetivos particulares a los que se anexa comunicación para -: desarrollo, salud, medioambiente, modernización rural, etc.; con lo que el paradigma vuelve a situar el cambio social en la posición dominante de la relación disciplinaria - para el cambio social_- y a la comunicación como un simple accesorio para catalizar un conjunto de mejoras. Además de acentuar el esquema hiper-especializado y fragmentador de las ciencias sociales, esta concepción errónea conduce a que aún no se haya tomado plena conciencia de la autonomía y el potencial híbrido de la comunicación para manejar lo específico del resto de disciplinas. En este sentido, consideremos imprescindible la labor de pensar comunicacionalmente otros ámbitos, una mirada que ayudaría a completar saberes en los que la perspectiva cultural o comunicacional está ausente o es prácticamente inexistente: psicología, sociología, ciencias políticas, economía, etc. En este razonamiento no sería pues una preposición de finalidad, teleológica y lineal — para - lo 
que uniría a ambos conceptos, sino una simple conjunción $-y-$, tal y como han ya entendido otras subdisciplinas que valoran adecuadamente el carácter transversal e interdisciplinar de la comunicación — ej. comunicación/educación o edu-comunicación; comunicación y género-: "El reto que tenemos hoy es generar un proceso interdisciplinar para que se generen auténticos híbridos coyunturales que puedan ayudar a responder a las demandas en un momento determinado" (Obregón a Barranquero, 2009).

\subsection{EL DESCUIDO DEL SUJETO EN EL MEDIO AMBIENTE}

La nueva propuesta de comunicación para el cambio social se planteó sin haber acometido una revisión profunda de la matriz funcionalista y evolucionista del concepto de cambio social, un cuestionamiento que ya se ha dado en otras disciplinas - en especial en la sociología - , pero no en las propuestas diseñadas en Bellagio y Cape Town ${ }^{4}$ :

Aunque muchos de los investigadores se esfuerzan por darles rumbos nuevos a estas palabras $[\ldots]$ y hacerlas políticamente correctas, también el concepto de cambio social proviene de la sociología funcionalista norteamericana. Incluso sus métodos de trabajo hay que rastrearlos [...] en los aportes de la sociología clásica y la psicología social que hicieron autores como Gabriel Tarde, Robert Merton y el propio Paul Lazarsfeld [...] No basta pues con acuñar nuevos términos, cuando lo que hay detrás es todo un recorrido en el que [...] la palabra cambio social comenzó a remplazar a otra que entró en desuso, pero [...] sin saber hasta qué punto su proyecto político e ideológico sigue conservando su raíz inicial.

(Bonilla, en Barranquero, 2009)

Por otro lado, al insistir en la base comunitaria de las transformaciones - cambio social ${ }^{5}$,- la noción pierde exactitud, ya que subsume en esta dinámica otros órdenes del desarrollo como el individual o el ambiental. En otras palabras, el comunitarismo al que apela la nueva noción desatiende los múltiples tejidos de que se compone la subjetividad humana, y al conminar a los diferentes grupos humanos a que "definan autónomamente su presente y su futuro", se olvida el hecho de que cualquier propuesta de desarrollo se topa inexorablemente con un marco global de amenazas a la reproducción de la vida sobre la tierra, frente a los cuales no vale cualquier trazado decidido en comunidad. Una de las vías de salida a este dilema pasaría por integrar la dimensión de la construcción individual del cambio y por abordar un diálogo profundo entre lo mejor de los valores del socia-

4 Efectivamente, el concepto de cambio social no es nuevo en la tradición filosófica y sociológica. La sociología del cambio social viene trabajando en él desde los años 40 del siglo pasado para analizar el proceso de movilidad en las estructuras de clase/estatus contemporáneas, o los diferentes factores que dan lugar a una progresiva división del trabajo y burocratización las sociedades industrializadas. Su óptica en exceso funcionalista ha ido orientada a promover los cambios a través del consenso, descuidando las variables del conflicto o del poder/saber (Foucault, 1997) que obstaculizan cualquier proceso dialógico.

5 Una visión deudora de los planteamientos de comunicación alternativa y participativa, de raíz latinoamericana desde la segunda mitad del siglo XX. 
lismo - justicia y equidad - y el liberalismo — libertad — (Alfaro, 2006: 47) ${ }^{6}$. En la variante ambiental, resulta necesario pensar la disciplina desde las bases de una nueva racionalidad ecológica (Leff, 1994; Maldonado, 1999), que nos aleje para siempre de una modernidad que creyó ciegamente en el progreso ilimitado de la tecno-ciencia a expensas de la naturaleza.

\subsection{EL RECICLAJE DEL ANTIGUO CONCEPTO DE DESARROLLO}

En tercer lugar, y lo que es más grave, el nuevo paradigma propone como horizonte utópico el cambio social, pero esta concepción finalista acaba reciclando y preservando la esencia misma del desarrollo. Cambio apunta siempre a un telos, a un fin o dirección, y éste nos remite de forma directa a la herencia occidental, evolucionista y mecanicista del antiguo concepto (Rist, 2002), así como a la senda del crecimiento económico, el aumento de la capacidad de consumo o la producción ilimitada:

Cualquiera que sea el adjetivo que se le dé, el contenido implícito o explícito del desarrollo es el crecimiento económico, la acumulación del capital con todos sus efectos positivos y negativos que conocemos: competencia sin piedad, crecimiento sin límites marcado por las desigualdades, pillaje sin reparar en la naturaleza. [...] Ese núcleo duro que todos los desarrollos tienen en común con esa experiencia está relacionado con 'valores' como el progreso, el universalismo, el dominio de la naturaleza, la racionalidad cuantificante. Esos valores sobre los que reposa el desarrollo y particularmente el progreso, no se corresponden en absoluto con aspiraciones universales profundas. Están relacionadas con la historia de Occidente, tienen poco eco en otras sociedades (Latouche, 2007: 21).

El nuevo paradigma de la comunicación para el cambio social apela a lo endógeno, pero preserva inconscientemente el trazado exógeno y universalista que tan trágicos resultados ha deparado sobre los recursos del planeta. Si una de las premisas del comunicador para el cambio es el respeto a la autonomía y diversidad de las culturas, no podemos seguir insistiendo en la idea de desarrollo o de progreso, sobre todo porque hay colectivos que no desean ni necesitan cambiar, sino mantener y consolidar sus antiguas estructuras. En cambio, otras culturas, las del capitalismo avanzado, están obligadas a decrecer o, al menos, a articular una relación más armónica con la naturaleza. En suma, el ser humano precisa de la comunicación y la cultura, no para evolucionar en dirección alguna, sino para pensar o articular antiguas y nuevas cosmovisiones y modos de vida, acordes con la solidaridad comunal y con la sostenibilidad de vida humana sobre la tierra.

\footnotetext{
6 En la misma línea se sitúan planteamientos críticos con el comunitarismo como los de Victoria Camps: "Una moral que se fundamenta sólo en el consenso comunitario es una moral tan heterónoma como la que descansa en el código religioso. No es la tribu la base de una posible moral pública, sino la aceptación por parte de cada individuo de su condición de ciudadano. [...] La división de la política en derechas e izquierdas, y la apropiación por la derecha de la ideología liberal, consiguió que una izquierda ya irreconocible desertara incluso de los valores liberales más imprescindibles” (1999, p. 23).
} 


\section{LA ARTICULACIÓN CON OTRAS DISCIPLINAS}

Una de las direcciones para enriquecer o incluso escapar del concepto de comunicación para el cambio social pasaría por entablar un diálogo más estrecho con un conjunto de sub-disciplinas que han venido articulando prolíficas reflexiones en los últimos años: la educación ambiental, la comunicación ambiental y los Estudios Culturales Verdes. Todas ellas se aproximan a la cuestión de la comunicación y la cultura como dimensiones integradas en la propia naturaleza, y, por ende, poco susceptibles de ser separadas: "La cultura no es nuestra naturaleza, no; la cultura es algo propio de nuestra naturaleza, y eso es lo que vuelve más difícil nuestra vida. La cultura no suplanta la naturaleza, sino que la suplementa" (Eagleton, 2001).

La educación ambiental (environmental education) ${ }^{7}$ es el área más veterana, puesto que sus primeros trazados surgen a finales de los años 60 (Stapp, 1969), con un reconocimiento oficial de su existencia a partir de la Conferencia de las Naciones Unidas sobre el Medio Humano (Estocolmo, 1972) ${ }^{8}$. A grandes rasgos, esta disciplina se puede definir como un campo de investigación orientado a implementar conocimientos, valores y destrezas para que individuos y grupos establezcan una relación más estrecha y sostenible con la naturaleza ${ }^{9}$. Aunque resulte complicado encuadrar bajo una misma etiqueta la extensa diversidad de planteamientos, existe un cierto acuerdo entre los especialistas al valorar que la educación ambiental es también educación para la acción, puesto que parte del análisis de los problemas socio-ambientales, para construir espacios colectivos de deliberación, toma de decisiones y actuaciones para la resolución de los problemas ecosociales presentes y futuros. La disciplina considera asimismo que los procesos de aprendizaje no pueden valorarse al margen de su relación con el entorno natural, por lo que relación educativa se desarrolla toda la vida - es decir, atañe tanto a procesos de educación formal como informal - y en contextos pedagógicos tan variados como la escuela, los medios de comunicación, el hogar, los espacios de ocio o de trabajo, etc. (Caride y Meira, 2001; Disinger, 1983; Novo, 2003).

Ligada a la anterior, aunque con un origen algo más tardío, se encuentra la comunicación ambiental (environmental communication) ${ }^{10}$, cuyos primeros funda-

\footnotetext{
7 A veces es posible encontrar referencias al mismo ámbito con etiquetas como las de pedagogía ambiental (environmental pedagogy), pedagogía verde (green pedagogy), educación para el medio ambiente (education for environment), etc.

8 En el documento emanado de esta conferencia se explicitaba por primera vez lo indispensable de la educación ambiental con el objeto de ensanchar el conocimiento de la opinión pública y mejorar las conductas ambientales (Principio 19). Posteriormente cabría destacar reuniones como las de Belgrado (1975) en la que se definen las primeras metas, objetivos y principios del área, Tbilisi (1977), en el que se acuerda la incorporación de la materia a los sistemas de educación y de cooperación internacional; Moscú (1987), de donde emana una estrategia internacional para la acción en la década de los 90; o en la Cumbre de la Tierra de Río de Janeiro (1992) en la que la Agenda 21 dedica su capítulo 36 a la capacitación ambiental.

9 Definición basada en la propuesta del Congreso Internacional de Educación y Formación sobre el Medioambiente (Moscú, 1987).

10 Al igual que en el caso de la educación ambiental, existen diferentes denominaciones para el área -información ambiental (environmental information), periodismo ambiental (environmental journalism)-, así como subdisciplinas directamente ligadas como la comunicación y riesgo, o los enfoques de comunicación y cambio climático.
} 
mentos datan de la década de los 80 , momento en el que las temáticas ambientales ascienden con fuerza en la agenda de los medios (Milsten, 2009: 345) y se alzan las primeras voces críticas contra un modelo mediático y publicitario que desatiende el impacto ecológico de sus acciones ${ }^{11}$. Al contrario de lo que se piensa, la comunicación ambiental tiene unos presupuestos más amplios que su predecesor, el periodismo ambiental, puesto que no está basada en la generación de mensajes medioambientales, sino que emana de la premisa de que los seres humanos, en sus acciones cotidianas, manejan recursos naturales sobre los que informan y toman decisiones. La disciplina estaría pues orientada a investigar la construcción comunicativa de los problemas ambientales, así como la negociación de las diferentes respuestas sociales a los mismos, ofreciendo un vehículo pragmático y normativo para nuestro mejor entendimiento y relación con el entorno natural (Cox, 2006). Dicho en otras palabras, la disciplina combina una perspectiva analítica - investigar lo que ocurrecon la intervención; o sea, no busca simplemente transmitir mensajes, sino conformar procesos que influyan en el cambio de actitudes, valores y prácticas para mejorar la calidad de vida humana sin comprometer el deterioro de la biosfera.

Algo más desconocida para los profesionales de la comunicación para el de-sarrollo es la tercera de las disciplinas emergentes, esta vez desde finales de la década de los 90: los Estudios Culturales Verdes, inspirada en la Ecocrítica -Ecocriticism-, más propia de los estudios literarios (Glotfelty y Fromm, 1996) ${ }^{12}$. Si atendemos a la etiqueta verde, esta disciplina estaría orientada al igual que su perspectiva matriz, los Cultural Studies, al estudio de los textos - escritos, audiovisuales, orales, digitales - que recogen la idea de lo natural o de la relación de lo humano con lo no humano, a la manera de autores como Donna Haraway o Bruno Latour. El campo intenta suplir la ausencia de la problemática ambiental en el ámbito de los Estudios Culturales, recuperando, por ejemplo, las reflexiones de autores de la tradición como Raymond Williams (1973, 1980), y haciendo dialogar la idea de lo natural con sus categorías habituales: poder, clase social, sexo, etnia o edad. Los Green Cultural Studies denuncian asimismo las representaciones culturales que participan de dicotomías jerárquicas propias del pensamiento ilustrado - cultura / naturaleza, materia / espíritu, mente / cuerpo, orgánico / inorgánico, etc.- y analizan ciertas concepciones sobre lo natural —ecología, ambientalismo, lo salvaje, lo pastoril, lo rústico, etc. ${ }^{13}$ - a los que consideran factores explicativos directos de la crisis cultural y ecológica que vive el planeta: "El proyecto de los estudios culturales verdes es el examen de la naturaleza a través de las palabras, la imagen $[\ldots]$ con el objetivo de poner en primer plano los potenciales efectos que esta representación podría tener sobre prácticas culturales que, alternativamente, afectan a la naturaleza" (Hochman, 1998). Además de una dimensión analítica, estos estudios cuen-

11 Es el caso, por ejemplo, de las espectaculares acciones de protesta de colectivos como Greenpeace de los años 80 .

12 La Ecocrítica también es conocida en algunos círculos como Crítica Literaria Ambiental (Environmental Literary Criticism), Crítica Verde (Green Criticism), Estudios Verdes (Green Studies), Ecopoética (Ecopoetics) o Literatura Ambiental (Environmental Literature).

13 Incluso desde una perspectiva autocrítica, los ECV intentan velar por los posibles efectos de sus propias construcciones discursivas sobre los imaginarios sociales, siguiendo los dictados de Michael P. Cohen: "Si quieres ser ecocrítico, prepárate para explicar lo que haces y para ser criticado, cuando no satirizado". 
tan con una faceta aplicada y normativa puesto que, en último término, se adentran en el diseño de nuevos modelos de representación y escritura natural — natural writing(Buell, 1995), de tipo artístico, cultural o incluso científico, en los que el entorno deja de considerarse mero recurso material o simbólico para concebirse como un todo sistémico en el que se enmarca la propia vida de los seres humanos.

De acuerdo a lo descrito, y aunque constituyen tradiciones de estudio autónomas, las tres disciplinas comparten algunas similitudes epistemológicas y metodológicas emparentadas con el campo de la comunicación para el desarrollo, y en especial con los planteamientos participativos más avanzados.

\subsection{SU ORIENTACIÓN INTERDISCIPLINAR}

Aunque en cada uno de los campos predomina una matriz disciplinar ${ }^{14}$, los tres se consideran materias transversales a otras y tienden a romper con la rigidez y la compartimentación propias de las ciencias sociales modernas y positivistas. De esta manera, piensan el binomio comunicación y medioambiente desde matrices tanto sociales y humanísticas como naturales y experimentales, y apuestan por lo interdisciplinar como fórmula epistémica para trazar puentes entre ciencia, cultura y medioambiente. En ellas se hacen difusos los límites entre lo cuantitativo y lo cualitativo, lo analítico y lo aplicado, lo empírico y lo ensayístico. Y más allá de la mirada reduccionista informacional o periodística, las tres disciplinas conciben la comunicación como un proceso a largo plazo - y no como un mero acto o un productode interrelación entre seres humanos, con mediación o no de tecnologías, y en un entorno en el que los medios no son más mediaciones interdependientes de un ecosistema a la vez mediático, cultural y físico.

\subsection{LA PERSPECTIVA CRÍTICA Y NORMATIVA Y EL COMPROMISO CON LA PRAXIS TRANSFORMADORA}

Las tres disciplinas apuestan por enfoques críticos emparentados con los presupuestos de Frankfurt, a saber: la ruptura de la falsa dicotomía positivista entre sujeto y objeto; el carácter histórico y diacrónico de la investigación; la orientación normativa y ética en torno a conceptos como sostenibilidad o responsabilidad ambiental; la vinculación entre teoría y praxis y la búsqueda del cambio social o de modelos de vida armónicos; o la apertura hacia perspectivas complejas y sistémicas que alertan acerca de la relación inseparable entre ser humano, ciencia, tecnología y medioambiente. Su perspectiva se sitúa además en un punto intermedio entre el ser y el deber ser de las prácticas culturales y ambientales; o lo que es lo mismo, entre el terreno de lo estrictamente observable y un horizonte ecosocial más equilibrado.

14 Las disciplinas principales de adscripción son la comunicación en el caso de la comunicación ambiental; la educación en la educación ambiental; y los estudios literarios y culturales en el caso de los Green Cultural Studies. 


\subsection{LA CRÍTICA A LOS MODELOS CULTURALES Y AMBIENTALES IMPERANTES}

Otra de las señas de identidad es el planteamiento de una crítica epistemológica profunda al modelo cultural dominante en campos como los de la economía o la política - en particular los planteamientos soft y ecológicamente maquillados de la economía convencional dominante y sus manifestaciones neoclásicas, liberales o de modernización ecológica-; esto es, la incitación de la publicidad al consumo insostenible sobre la base de la obsolescencia programada o la difusión mediática de modos de vida poco acordes con los ecosistemas. Las disciplinas incorporan además una reflexión profunda acerca de la construcción moderna y eurocéntrica de los saberes, incorporando variables como el poder o el conflicto y partiendo de la consideración de que no existen verdades naturales o esenciales, sino que saber y poder están estrechamente ligados, a la manera que advirtió Foucault: "Lo que le da estabilidad al poder, lo que induce a tolerarlo, es el hecho de que no actúa solamente como una potencia que dice no, sino que también atraviesa las cosas, las produce, suscita placeres, forma saberes, produce discursos" (Foucault, 1998). Las tres disciplinas invitan, en suma, a plantear una genealogía de los saberes que desvele las relaciones de desequilibrio científicas, geográficas Norte/Sur, de clase, etnia, género, etc.- en el ámbito de las ciencias, y contribuya a descentralizar el conocimiento moderno y a superar la fractura entre saberes formalizados y tácitos - saberes ecológicos tradicionales- o entre perspectivas euro-céntricas y saberes periféricos y coloniales.

Llegados a este punto, también conviene advertir de que ya se están dando los primeros pasos para el diálogo entre la comunicación para el cambio social y el resto de disciplinas. Este es el caso, por ejemplo, de analistas que incitan a una reconversión de nuestra materia desde una perspectiva ecológica, ya sea a partir de denominaciones como las de comunicación para el desarrollo sostenible (Servaes y Malikhao, 2007; Mefalopulos, 2005), ya sea aproximando la dirección a otras dimensiones como el empoderamiento ecosocial (Chaparro, 2009b) o la comunicación para la sostenibilidad (Díaz Nosty, 2009; Gumucio Dagron, 2006). Asimismo, son interesantes los trabajos sobre experiencias prácticas en el terreno como los de Flor (2004) o Herrera (2011).

\section{COMUNICACIÓN, JUSTICIA ECOSOCIAL Y BUEN VIVIR}

Otra de las vías de salida para los dilemas que afronta nuestra disciplina es entablar puentes con conceptos que animan a descartar, de una vez por todas, la dirección marcada por el concepto del desarrollo, a partir de una crítica al mismo y desde la apertura a otras dimensiones de la vida humana que no tienen que ver con lo acumulativo, lo finalístico o lo universalista. Nos referimos a prolíficas nociones como justicia ecosocial o buen vivir, que, aunque ya cuentan con algunos desarrollos en campos como la sociología, la ecología, el derecho o la economía, aún no han permeado lo suficiente a la comunicación, si bien su enfoque es por definición interdisciplinario, crítico y normativo, en la línea de las reflexiones más avezadas de nuestra materia. 
En primer lugar, conviene destacar la prolífica línea de investigación abierta en torno a la idea fuerza de justicia social y otras nociones anexas como las de exclusión moral (Opotow, 1990), homo sacer (Agamben, 1998) o justicia ecológica (Gudynas, 2010), que ya cuentan con implementaciones - especialmente la primera - en el ámbito de la comunicación, aunque los intentos han sido por el momento coyunturales y carentes de un marco teórico común (Frey, 1998; Leets, 2001; Jansen, Pooley y Taub-Pervizpour, 2011).

Pese a que la idea de justicia social aparece en el siglo XIX en la obra de autores como Weber, Mill o Marx (Leets, 2001; Morris, 2009), su traslación al campo de la sociología y la política datan de finales del siglo pasado. Ésta se entiende ya no desde el campo jurídico propiamente dicho, sino desde un ideal de justicia distributiva (Rawls, 1979), que se aleja de la concepción clásica de justicia entre iguales para reclamar un reparto justo de los bienes y recursos de los que los excluidos se suelen ver privados, en base a un análisis previo de sus necesidades e intereses. En otras palabras, esta noción parte de un diagnóstico de la desigualdad social y la exclusión moral (Opotow, 1990), frente a la cual se propone un nuevo marco de derechos y políticas públicas.

En esta línea, uno de los autores más reconocidos en nuestro campo ha sido el economista indio Amartya Sen que, en textos como Desarrollo y Libertad (2000) o La idea de justicia (2010) ${ }^{15}$, promueve lo que él denomina el enfoque de las capacidades básicas (capability), o del desarrollo como libertad. Para Sen, el desarrollo no es más que una implementación de la libertad como medio y sobre todo como fin, teniendo en cuenta que "la eficacia de la libertad como instrumento reside en el hecho de que los diferentes tipos de libertad están interrelacionados y un tipo de libertad puede contribuir extraordinariamente a aumentar otros" (Sen, 2000: 56). Siguiendo este razonamiento, las políticas públicas de gobiernos y organizaciones han de promover capacidades reales (capabilities) para que los ciudadanos dispongan de libertad para decidir la forma en la que quieren vivir. Así, el poder de crear riqueza ya no equivale a acumulación económica, sino a habilitar un conjunto de prerrequisitos para la obtención de libertad o justicia social, tales como, en comunicación, la eliminación de barreras a la discusión libre y abierta, la institucionalización del derecho a la comunicación, o la instauración de unos medios saludables y sin restricciones para dar voz a los desprotesgidos.

Sin ser desdeñables estos aportes, resultan mucho más completas aquellas visiones que, desmarcándose de la herencia de Rawls, visible en Amartya Sen, apuestan por reconocer que la propia naturaleza es sujeto de derechos. Esta concepción ya no parte de la herencia moderna de atribuir racionalidad o moralidad a la naturaleza, sino de la consideración de que los seres vivos tienen derecho a disfrutar de su desarrollo como tales y de que todas las formas de vida son mutuamente interdependientes entre sí y de un soporte físico (Baxter, 2005; Gudynas, 2010; Leff, 2001; Low y Gleeson, 1998; Scholsberg, 2009).

15 Las nociones de Amartya Sen han tenido un desarrollo incipiente en algunos de los textos que atañen al área, retomados por investigadores del campo de la comunicación participativa (Gumucio-Dagron, 2010; Servaes y Malikhao, 2007), 
Esta idea de justicia ecológica —o de manera más completa de justicia ecosocial-, se alía asimismo con conceptos que, a diferencia de los anteriores, nacen en la propia "periferia social de la periferia mundial" (Tortosa, 2009:3) y se alejan de la dirección trazada por el programa colonial del desarrollo. Uno de los más interesantes al respecto, ausente aún en el ámbito de la comunicología, es la noción del Sumak Kawsay — o Suma Qamaña, en aymara- (Acosta y Martínez, 2009), reivindicada por las culturas ancestrales indígenas de Ecuador y Bolivia e incorporada a las Constituciones de los respectivos países en 2008 y 200916, aunque por el momento no hayan sido implementadas in extenso en las políticas y legislaciones que derivan de estos marcos constituyentes.

Para comprender lo que significan estas nociones, conviene recordar que en muchas de las cosmovisiones indígenas de la región andina no existe si quiera la concepción de un proceso lineal del desarrollo o de un estado de subdesarrollo a ser superado o de desarrollo por alcanzar. Por otro lado, la propia concepción de pobreza o subdesarrollo tampoco se suele asociar a la carencia de bienes materiales, de igual manera que la riqueza no se vincula a la abundancia de estos (Acosta, 2008: 34). La categoría tiene más que ver con el concepto clásico griego de vida buena (Robinson y Lefka, 2009) o, en su concepción del aymará boliviano, Suma Quamaña, de una vida buena que introduce un elemento comunitario y solidario y que se podría traducir como buen vivir, o buen convivir en armonía (Totosa, 2009: 1). Lo que está en juego aquí es la vida misma (Acosta, 2008: 34) o la vida en plenitud (Dávalos, 2011: 201); es decir, una concepción que desborda los límites de un proyecto meramente económico, social o político, y que adquiere el carácter de paradigma regulador del conjunto total de la vida tanto social como natural.

De hecho, en esta perspectiva holística, los bienes materiales ya no son tan determinantes como cuestiones como el "conocimiento, el reconocimiento social y cultural, los códigos de conductas éticas e incluso espirituales en la relación con la sociedad y la naturaleza, los valores humanos, la visión de futuro, entre otros" (Acosta, 2008: 34). Y frente a lo inviable de los modelos de (comunicación para) el desarrollo dominantes, el Sumak Kawsay parte de saberes ecológicos tradicionales y vivencias ancestrales de los pueblos indígenas como forma de resistencia al capitalismo y la modernidad a partir de la incorporación de nuevos modos de socialidad basados en un profundo respeto y convivencia con la naturaleza:

El concepto de Sumak Kawsay permite precisamente esto: una nueva visión de la naturaleza, sin desconocer los avances tecnológicos ni los avances en la productividad, sino más bien proyectándolos al interior de un nuevo contrato con la naturaleza, en la que la sociedad no se separe de ésta, ni la considere como algo externo, o como

\footnotetext{
16 Además de que en las respectivas constituciones se reconoce a la naturaleza (o Pacha Mama) como sujeto portador de derechos, el Sumak Kawsay ha sido incorporado en la Constitución de Ecuador de 2008, en la que "se reconoce el derecho de la población a vivir en un ambiente sano y ecológicamente equilibrado, que garantice la sostenibilidad y el buen vivir, sumak kawsay" y la Carta Constituyente de Bolivia, en la que, entre otros principios ético-morales de la sociedad plural se incluye la Suma Quamaña (buen vivir) o conceptos como ñandereko (vida armoniosa), teko kavi (vida buena), etc.
} 
una amenaza, o como otro radical, sino como parte de su propia dinámica, como fundamento y condición de posibilidad de su existencia a futuro (Dávalos, 2011: 209).

En la línea de las reflexiones de la justicia ecológica, el concepto contribuye a problematizar la pobreza y la exclusión ya no en términos materiales, como en la valoración de los contextos de poder en los que la pobreza económica es expresión del control político de la escasez. En otros términos, ya no se trataría de resolver la pobreza con los instrumentos del propio capitalismo, sino que sería necesario alentar un cambio o sustitución radical del propio sistema capitalista, abocado a generar una pobreza que "está en su mismo formato, en su misma episteme" (Ibíd.: 212).

En esta línea el Sumak Kawsay apunta a la necesidad de retomar planteamientos fecundos que apuestan por una reconversión radical del propio sistema capitalista neoliberal, en torno a ideas como las de decrecimiento, el slow life, el respeto a la biomímesis, o vivir más con menos, que abanderan pensadores herederos de los postulados de Nicholas Georgescu-Roegen (2001) como Serge Latouche (2007), Enrique Leff (1994), Joan Martínez Alier (2005), José Manuel Naredo (2006) o Jorge Riechmann (2011).

\section{CONCLUSIONES}

Parafraseando uno de los textos más conocidos del edu-comunicador argentino Mario Kaplún, A la educación por la comunicación (1992), el siguiente estudio ha procurado problematizar algunas de las principales fallas de la pregunta que hasta hoy ha guiado el devenir del campo, ya sea en su acepción neodifusionista o participativa: ¿cómo emplear los medios de comunicación para introducir desarrollo en la sociedad? Si pensamos la disciplina como un área de investigación y acción en torno al binomio dialéctico comunicación-justicia ecosocial y buen vivir, o, en otras palabras, si nos aventuramos a dar un giro al orden mismo del enunciado - icómo pensar la justicia ecosocial y la vida buena desde la comunicación y la cultura?-, estaremos avanzando hacia los fundamentos de una ciencia nueva, que estará por lo demás:

- No sólo centrada en los medios y tecnologías de apoyo al desarrollo, sino, sobre todo, en cómo articular desde la comunicación procesos culturales, sociopolíticos o económicos con voluntad de cambiar el injusto orden de cosas, sin una concepción meramente lineal y acumulativa, o exclusivamente basada en medios y tecnologías, sino en la recuperación de la palabra invisibilizada o en la articulación de nuevos lenguajes de valoración (Martínez Alier, 2005), estéticas, imaginarios, cosmovisiones o modos de vida.

- No orientado al diseño de metodologías universalistas del sistema de la cooperación en torno a indicadores desde entes supuestamente desarrollados a otros en vías de desarrollo. En su lugar, planteamos una ciencia híbrida basada en el quehacer y la reflexión menos institucionalizada de las prácticas de base, los saberes oriundos o el media-activismo transformador, que incorporan la multiplicidad de facetas de la vida mejor — socialidad, solidaridad, diversidad, derechos humanos, justicia ecológica, etc.—, tal y como están demostrando determinados estallidos sociales de nuevo cuño que emplean la 
comunicación participativa para reflexionar acerca del buen vivir y la justicia: 15-M, movimiento Occupy, etc.

- No basado en el desarrollo antropocéntrico del ser humano a expensas de la naturaleza, sino en una concepción biocéntrica del cambio ecosocial que supere las falsas dicotomías entre cultura y naturaleza, hombre y entorno, o desarrollo humano y desarrollo sostenible.

En resumen, la comunicación y el buen vivir no es sólo una disciplina sino un programa de reconversión radical de los saberes construido sobre las ruinas del proyecto de una razón moderna antiecológica y depredadora. La comunicación para el cambio social volverá a ser inoperante si no se cuestionan sus bases mismas hasta desecharla e incorporar sendas nuevas, tal vez siguiendo propuestas como las de la hermenéutica diatópica, de Boaventura de Sousa Santos (2005), que nos ayudan a comprender la incompletud intrínseca de cualquier cultura y nos aconsejan compartir nuestros discursos y proyectos con los de otras culturas, con el fin de regenerarlos y perfeccionarlos. Esta vía garantiza que hay múltiples caminos para la vidamejor, pero para salir a su encuentro no existe otra fórmula que la de la comunicación intercultural y el diálogo como medida central para la reinvención de un presente precario e incierto. Por otra parte, es necesario comenzar a enfrentar el devenir del Sur Global sobre la contención y desaceleración del Norte, puesto que existen pruebas irrefutables de que el modelo liberal globalizado nos está conduciendo de forma inexorable al accidente global. De esta manera, habrá que seguir confrontando la investigación colonial, evolucionista y lineal de la que nace la comunicación para el desarrollo, con nuevas concepciones acerca del buen vivir, así como con nuestra dependencia del entorno natural y el universo de riesgos ambientales globales que actualmente acechan.

\section{REFERENCIAS BIBLIOGRÁFICAS}

AcostA, Alberto (2008): "El buen vivir, una oportunidad por construir". Revista Ecuador Debate, 75, pp. 33-48.

Acosta, Alberto y MARTínez, Esperanza (comp.) (2009): El buen vivir. Una vía para el desarrollo. Santiago: Editorial Universidad Bolivariana.

Agamben, Giorgio (1998): Homo sacer. El poder soberano y la vida nula. Valencia: Pre-Textos.

Alfaro, Rosa María (2006): Otra brújula. Innovaciones en comunicación y desarrollo. Lima: Calandria.

BARRANQUERO, Alejandro (2009): Latinoamérica en el paradigma participativo de la comunicación para el cambio. Málaga: Universidad de Málaga.

- (2011a): "El espejismo de la comunicación para el cambio social. Radiografía de un concepto insostenible. Hacia una comunicación de cambio ecosocial". En J. M. Pereira y A. Cadavid (eds.) (2011): Comunicación, desarrollo y cambio social: Interrelaciones entre comunicación, movimientos ciudadanos y medios. Bogotá: Pontificia Universidad Javeriana, Universidad Minuto de Dios, UNESCO, FESCOL. 81-100.

(2011b): "Estudios culturales verdes. Una lectura ecocrítica de las relaciones entre cultura y medioambiente". En S. Álvarez (coord.): Convivir para perdurar. Conflictos ecosociales y sabidurías ecológicas. Barcelona: Icaria. 187-200. 
BAXTER, Brian (2005): Theory of ecological justice. Londres: Routledge.

BuELl, Lawrence (1995): The Environmental Imagination: Thoreau, Nature Writing, and the Formation of American Culture. Cambridge, London: Harvard University Press.

CAMPS, Victoria (1999): Paradojas del individualismo. Barcelona: Crítica.

CARIDE, José Antonio y MeIrA, Pablo Ángel (2001): Educación ambiental y desarrollo humano. Barcelona: Ariel.

Chaparro, Manuel (2009a): "Comunicación y desarrollo: retos para un nuevo periodismo". Telos, 81, pp. 27-40.

Chaparro, Manuel (2009b): Comunicación para el empoderamiento y comunicación ecosocial. La necesaria creación de nuevos imaginarios". Perspectivas de la comunicación, 2 (1), pp. 146-158.

Cimadevilla, Gustavo (2004): "Información ambiental, medios y actores: escenas de una desconexión anunciada" en Comunicación, tecnología y desarrollo. Córdoba: Universidad Nacional de Río Cuarto.

Cox, Robert (2006): Environmental Communication and the Public Sphere. Thousand Oaks: Sage Publications.

DÁvalos, Pablo (2011): “Sumak Kawsay (La vida en plenitud)”. En S. Álvarez (coord.): Convivir para perdurar. Conflictos ecosociales y sabidurías ecológicas. Barcelona: Icaria. 201-210.

DíAZ Nosty, Bernardo (2009): “Cambio climático, consenso científico y construcción mediática. Los paradigmas de la comunicación para la sostenibilidad”. Revista Latina de Comunicación, 64.

Disinger, John F. (1983): Environmental education's definitional problem. Columbus, OH: ERIC/CSMEE.

EAGLETON, Terry (2001): Cultura y naturaleza. Barcelona: Paidós.

FLOR, Alexander G. (2004): Environmental communication: Principles, approaches and strategies of comunication applied to environmental management. Quenzon: University of the Philippines. Open University.

Foucault, Michel (1988): Nietzsche, la genealogía y la historia. Valencia: Pre-Textos. (1998): La voluntad de saber. Madrid: Siglo XXI.

Georgescu-Roegen, Nicholas (2001): Ensayos bioeconómicos. Madrid: Catarata.

Gray-Felder, Denise y Deane, James (1999): Communication for Social Change: A Position Paper and Conference Report. Nueva York: Rockefeller Foundation.

GUDYNAS, Eduardo (2010): "La senda biocéntrica: valores intrínsecos, derechos de la naturaleza y justicia ecológica". Tabula Rasa, 13, pp. 45-71.

Gumucio-Dagron, Alfonso (2001): Haciendo olas. Historias de comunicación participativa para el cambio social. Nueva York: The Rockefeller Foundation.

(2007): El desafio de Babel. Etcétera. Para entender a los medios. 1 de enero.

Gumucio-Dagron, Alfonso y Tufte, Thomas (eds.) (2006): Communication for Social Change Anthology: Historical and Contemporary Readings. New Jersey, SO, Communication for Social Change Consortium.

Glotfelty, Cheryll y Fromm, Harold (eds.) (1996): The Ecocriticism Reader: Landmarks in Literary Ecology. Georgia \& London: University of Georgia. 
JANSEN, Sue Curry (2011): "Media, democracy, human rights, and social justice". En S. C. Jansen, J. Pooley y L. Taub-Pervizpour (eds.). Media and social justice. New York: Palgrave Macmillan.

GUDYNAS, Eduardo (2010): "La senda biocéntrica: valores intrínsecos, derechos de la naturaleza y justicia ecológica". Tabula Rasa, 13, pp. 45-71.

HERRERA, Eliana (ed.) (2011): Experiencias de comunicación y desarrollo sobre medio ambiente. Estudios de caso e historias de vida en la región andina de Colombia. Bogotá: UNAD, Uniminuto, USTA y Manuskripta.

Hochman, Jhan (1998): Green Cultural Studies. Nature in Film, Novel and Theory. Moscow, ID: University of Idaho Press.

Latouche, Serge (2007): Sobrevivir al desarrollo. De la descolonización del imaginario económico a la construcción de una sociedad alternativa. Madrid: Icaria.

LEETS, Laura (2001): "Interrumpting the cycle of moral exclusion: A communication contribution to social justice research". Journal of Applied Social Psychology, 31 (9), pp. 1859-1891.

LEFF, Enrique (1994): Ecología y capital: racionalidad ambiental, democracia participativa y desarrollo sustentable. México: Siglo XXI.

Low, Nicholas y GLEESON, Brendan (1998): Justice, society and nature. An exploration of political ecology. Londres: Routledge.

MALDONADO, Tomás (1999): Hacia una racionalidad ecológica. México: Infinito.

MARIÑO, Miguel Vicente (2011): "La expansión de la investigación sobre comunicación medioambiental: ¿otra consecuencia más del cambio climático?”. En S. Álvarez (coord.): Convivir para perdurar. Conflictos ecosociales y sabidurías ecológicas. Barcelona: Icaria, pp. 371-386.

Martínez Alier, Joan (2005): El ecologismo de los pobres. Conflictos ecológicos y lenguajes de valoración. Barcelona: Icaria.

Mefalopulos, Paolo (2005): "Communication for sustainable development: applications and challenges". En O. Hemer y T. Tufte (eds.). Media and glocal change: Rethinking communication for development. Buenos Aires: CLACSO. 247-259.

Milsten, Tema (2009): "Environmental communication theories". En S. W. Littlejohn y K. A. Foss (Eds.). Encyclopedia of communication theory. London: Sage.

Morris, Martin (2009): "Social justice and communication: Mill, Marx and Habermas". Social Justice Research, 22 (1), pp. 134-55.

NAREDO, José Manuel (2006): Raíces económicas del deterioro ecológico y social. Más allá de los dogmas. Madrid: Siglo XXI.

Novo, María (2003): La educacion ambiental: bases eticas conceptuales y metodológicas. Madrid: Universitas.

Оротоw, Susan (1990): “Deterring Moral Exclusion”. Journal of Social Issues, 46. 173-182.

RaWLs, John (1979): Teoría de la justicia. México: Fondo Cultura Económica.

RIsT, Gilbert (2002): El desarrollo: historia de una creencia occidental. Madrid: IUDC-La Catarata.

Servaes, Jan y Malikhao, Patchanee (2007): "Comunicación y desarrollo sostenible". En AA.VV.: Comunicación y desarrollo sostenible. Selección de artículos de la 9na mesa redonda de las Naciones Unidas sobre comunicación para el desarrollo. Roma: FAO. Pp. 1-42.

SEN, Amartya (2000): Desarrollo y libertad. Barcelona, Planeta. - (2010): La idea de la justicia. Madrid: Taurus. 
Scholsberg, David (2009): Defining environmental justice. Theories, movements, and Nature. New York: Oxford University Press.

Sousa SANTOS, Boaventura de (2005): El milenio huérfano: ensayos para una nueva cultura política. Madrid: Trotta.

STAPP, William B. (1969): “The concept of environmental education". Journal of Environmental Education, 1(1). pp. 30-31.

RieCHMANN, Jorge (2011): ¿Cómo vivir? Acerca de la vida buena. Madrid: Los Libros de la Catarata. TortosA, José María (2009): "Sumak Kawsay, Suma Quamaña, Buen Vivir”, en Seminario "Sumak Kawsay, aprendiendo del Sur". Alicante, 28-29 mayo.

Williams, Raymond (1973): The Country and the City. London: Chatto \& Windus, London. (1980): "Ideas of Nature". En Problems in Materialism and Culture: Selected Essays. London: Verso. pp. 67-85.

\title{
RESUMEN
}

El nuevo paradigma de la comunicación para el cambio social es una construcción en exceso comunitarista que recicla la herencia occidental, antropocéntrica y lineal del antiguo concepto de comunicación para el desarrollo. Las siguientes líneas se orientan a cuestionar las bases de esta nueva propuesta invitando a entablar un diálogo con un conjunto de prolíficas perspectivas como la educación y la comunicación ambiental o los Estudios Culturales Verdes. En último término, se intenta tender puentes hacia las nuevas nociones de justicia ecosocial y buen vivir como vías de reflexión para la construcción de una disciplina atenta al universo de riesgos globales y a la dependencia del ser humano y su cultura con respecto al entorno físico y natural.

Palabras clave: Comunicación para el desarrollo, comunicación para el cambio social, comunicación participativa, justicia social, justicia ecológica, comunicación ambiental, educación ambiental

\begin{abstract}
The new paradigm of communication for social change is a communitarist perspective that recycles the Western, anthropocentric and linear view of communication for development. This paper questions the roots of the new proposal by proposing a dialog with several prolific perspectives such as environmental education and communication or Cultural Green Studies. It also tries to build connections with the new notions of socio-environmental justice and good life as the right directions in order to build a discipline that faces the universe of global risks and human dependence with regard to physical and natural environment.

Key words: Communication for development, communication for social change, participative communication, social justice, ecological justice, environmental communication, environmental education
\end{abstract}

\section{RÉSUMÉE}

Le nouveau paradigme de la communication pour le changement social est une construction en excès communautariste qui recycle l'Occident, héritage anthropocentrique et linéaire de l'ancien concept de communication pour le développement. Les lignes suivantes visent à remettre en question les fondements de cette nouvelle proposition invitant à établir un dialogue avec un ensemble de perspectives prolifiques comme l'éducation et de la communication environnementale ou les études culturelles verts. En fin de compte, essayer de construire des ponts vers les nouvelles notions de Justice écosocial et bon vivante comme traces de réflexion pour la construction d'une discipline qui est attentive à l'univers des risques globaux et la dépendance sur les humains et leur culture avec ce qui a trait à l'environnement physique et naturel.

Mots clé: Communication pour le développement, communication pour le changement social, communication participative, justice sociale, justice écologique, communication environnementale, éducation environnementale. 